\section{P155 (continued)}

evaluation ensured theory-based learning outcomes were properly implemented and participants were reached.

Target Audience: Twelve first year college students (67\% female) enrolled at a large southeastern US university. Most (67\%) identified as non-Hispanic White.

Course/Curriculum Description: The curriculum was used for a pilot 12-week 1-credit hour exploratory course which included guest speakers from various food system sectors, case studies, documentary viewings, a cooking demonstration, and a culminating group project. The class met 1 evening per week and students were required to receive a satisfactory grade ( $70 \%$ or higher) to receive credit.

Evaluation Methods: Dose delivered was evaluated by curriculum implementation and instructor effectiveness. Student engagement and likability evaluated dose received. Class attendance assessed reach, and fidelity was evaluated by curriculum completion and course implementation alignment of the theoretical framework and learning outcomes. Context was assessed via situational factors.

Results: Dose delivered was high with $92.86 \%$ of the implementation checklist met. Dose received was adequate with a score of $3.80 \pm 0.63$ out of 5 for engagement and $4.13 \pm 0.28$ out of 5 for likability. Reach was high with a class mean attendance of $11.20 \pm 0.68$ students. Fidelity was considered high with $100 \%$ completion reported for the curriculum checklist and high alignment of the theoretical framework and learning outcomes. Context assessment revealed external barriers prohibited lessons to be taught chronologically but did not impact curriculum delivery.

Conclusions: Well-developed food systems education may shape future sustainable food practices in college students. Process evaluation of this pilot course suggested overall adequate implementation and future scaled-up testing was deemed an appropriate next step.

Funding: NIFA.

\section{P156 Impact of Culinary Nutrition Education on Student's Knowledge and Ability to Make Culinary Recommendations for the DASH-Diet}

Carmen Oh, BS, San José State University; Jamie Kubota, MS, RD, jamie.kubota@sjsu.edu, San José State University, One Washington Square, San José, CA, 95192; Colette LaSalle, $P h D, R D$, San José State University

Background: Dietetics students receive DASH-diet education as part of their medical nutrition therapy (MNT) curriculum. The ability for students to translate that knowledge into practice is currently unknown. There is evidence supporting that culinary nutrition education integrated into coursework has shown a positive association with nutrition knowledge and self-efficacy in students, adults, and health professionals. However, there is limited research showing the impact of culinary nutrition education on dietetics students.
Objective: To determine the impact of a combined lecture and DASH-diet culinary module for undergraduate dietetics students on knowledge and self-efficacy in making culinary-related recipe adaptations.

Study Design, Setting, Participants: This is a quasiexperimental pre-, post-study design. Using a non-probability and purposive sampling technique, students were recruited from a MNT class at San José State University (SJSU) and were randomly assigned to control $(\mathrm{n}=12)$ and intervention $(\mathrm{n}=13)$ group. All students received a DASHdiet lecture while only the intervention group received a culinary module following the lecture.

Measurable Outcome/Analysis: Data collection involved a questionnaire administered pre- and postintervention and during a 1-month follow-up. Data analysis included descriptive statistics reported as means compared between and within groups using SPSS version 25.

Results: There was a statistically significant improvement in identifying maximum amount of sodium in post $(P=.009)$ and ingredient substitutions in pre $(P=.12)$ and post $(P=.008)$ between control and intervention group. There was a statistically significant improvement in identifying sodium-containing ingredients $(P=.046)$ and ingredient substitution $(P<.00)$ within the intervention group.

Conclusions: This pilot study shows culinary nutrition education has the potential to positively impact dietetics students' knowledge on the DASH diet. Future programs should incorporate longer modules with additional opportunities to engage in hands-on practice and promote knowledge retention.

Funding: None.

\section{P157 Undergraduate Nutrition Students Develop Nutrition Counseling Skills Through a Healthcare Theatre Program}

Emily Nowosielecki, BS, RDN, University of Delaware; Sandra Baker, EdD, LDN, RDN, bakers@udel.edu, University of Delaware, 023 Carpenter Sports Building, Newark, DE, 19716; Anja Leefeldt, MS, LDN, RDN, University of Delaware

Objective: To evaluate the effectiveness of a Healthcare Theatre Program in developing counseling skills in nutrition students

Use of Theory or Research: Simulations can provide undergraduate nutrition students with experiences that develop their counseling skills, preparing them for their internships and careers. The Transformative Learning Theory maintains problem solving and self-reflection help students transform into clinicians.

Target Audience: A total of 404 nutrition students who participated in the Healthcare Theatre Program from 2013-2019.

Course/Curriculum Description: A Healthcare Theatre Program, as part of a nutrition counseling class, offers a unique simulation experience for students to develop 